\title{
RNA transport during TMV cell-to-cell movement
}

\section{Eduardo J. Peña ${ }^{1}$ and Manfred Heinlein ${ }^{1,2 *}$}

1 Institut de Biologie Moléculaire des Plantes du Centre National de la Recherche Scientifique, Université de Strasbourg, Strasbourg, France

${ }^{2}$ Department of Plant Physiology, University of Basel, Basel, Switzerland

\section{Edited by:}

Yiguo Hong, Hangzhou Normal

University, China

Reviewed by:

Sergey Morozov, Moscow State

University, Russia

Yule Liu, Tsinghua University, China

Lesley Torrance, The James Hutton Institute, UK

John Carr, Cambridge University, UK

\section{*Correspondence:}

Manfred Heinlein, Institut de

Biologie Moléculaire des Plantes du

Centre National de la Recherche

Scientifique, UPR 2357, Université

de Strasbourg, 12, Rue du Général

Zimmer, 67084 Strasbourg cedex,

France.

e-mail: manfred.heinlein@ibmp-

cnrs.unistra.fr
Studies during the last 25 years have provided increasing evidence for the ability of plants to support the cell-to-cell and systemic transport of RNA molecules and that this process plays a role in plant development and in the systemic orchestration of cellular responses against pathogens and other environmental challenges. Since RNA viruses exploit the cellular RNA transport machineries for spreading their genomes between cells they represent convenient models to investigate the underlying mechanisms. In this regard, the intercellular spread of Tobacco mosaic virus (TMV) has been studied for many years. The RNA of TMV moves cell-to-cell in a non-encapsidated form in a process depending on virus-encoded movement protein (MP). Here, we discuss the current state of the art in studies using TMV and its MP as a model for RNA transport. While the ability of plants to transport viral and cellular RNA molecules is consistent with RNA transport phenomena in other systems, further studies are needed to increase our ability to visualize viral RNA (VRNA) in vivo and to distinguish RNA-transport related processes from those involved in antiviral defense.

Keywords: Tobacco mosaic virus, plasmodesmata, RNA transport, RNA labeling, endoplasmic reticulum, cytoskeleton, movement protein, replication complex
Tobacco mosaic virus (TMV) is a rod-shaped virus with a positivesensed RNA genome that encodes $126 \mathrm{kD}$ and $183 \mathrm{kD}$ subunits of replicase, a $30 \mathrm{kD}$ movement protein (MP) and a $17.5 \mathrm{kD}$ coat protein (CP). The virion consists of the viral RNA (vRNA) protected by 2130 subunits of assembled CP. The virus has been a paradigm for RNA virus movement since the requirement of its MP for virus movement was defined (Deom et al., 1987; Citovsky, 1999; Waigmann et al., 2007). In addition, the virus provides a valuable tool to study RNA transport as the spread of infection does not require CP and the vRNA moves cell-to-cell in a nonencapsidated form. The MP has sequence-nonspecific binding affinity to single-stranded nucleic acids in vitro (Citovsky et al., 1990) and likely forms a ribonucleoprotein (vRNP) complex with vRNA upon replication in vivo. Based on experimental evidence suggesting that also the replicase is involved in virus movement (Hirashima and Watanabe, 2001; Guenoune-Gelbart et al., 2008) and that the virus moves in the form of replication complexes (Kawakami et al., 2004), the vRNP may be associated with several viral and host proteins in addition to vRNA and MP.

\section{vRNA TRANSPORT OCCURS VIA THE ER/ACTIN NETWORK}

To facilitate the cell-to-cell spread of this large vRNP, the MP increases the size exclusion limit of plasmodesmata (PD) (Wolf et al., 1989; Oparka et al., 1997), the intercellular communication channels in the plant cell walls (Heinlein, 2002; Heinlein and Epel, 2004; Maule, 2008). Although the virus may spread into adjacent cells from replication sites established near the channels (Szécsi et al., 1999; Kotlizky et al., 2001), the spread of infection through whole tissues depends on the transport of the virus across the diameter of the cells (Figure 1). Thus, from PD used for cellular entry the vRNP first binds to intracellular sites to establish new viral replication complexes (VRC) and to amplify vRNA. And, in a second step, the newly replicated vRNPs are transported from VRCs to the PD that link the infected cell to neighboring, yet non-infected cells. One important mechanism that may contribute to virus transport is cytosplasmic streaming (VerchotLubicz and Goldstein, 2010). Despite the large size of the vRNP, this process may contribute to distributing the vRNPs around in the newly entered cells before they attach to new sites for replication. However, once replicated, the new vRNP particles must be specifically targeted from the replication sites to PD, which likely depends on more specialized mechanisms. Consistent with this potential requirement for transport to $\mathrm{PD}$, numerous studies highlighted the importance of MP associations with dynamic endoplasmic reticulum (ER) membranes and components of the cytoskeleton (Heinlein et al., 1995, 1998; Boyko et al., 2000a, 2007; Brandner et al., 2008; Sambade et al., 2008) (for review, see Niehl and Heinlein, 2011; Peña et al., 2012). According to the current model (Figure 1), infection of a new cell starts with the association of the vRNP with sites on the ER. Translation and replication of the vRNA leads to the formation of distinct, ERlocalized VRCs that increase in size over time and finally form so-called X-bodies that produce virus progeny. Just following infection of the new cell and before the VRCs increase in size, the virus already moves further into adjacent cells, presumably in the form vRNP- containing VRCs or VRC sub-complexes that detach from their ER anchorage sites for subsequent transport via lateral diffusion along the ER membrane. Since the ER network is contiguous between cells through PD (Maule, 2008) it 


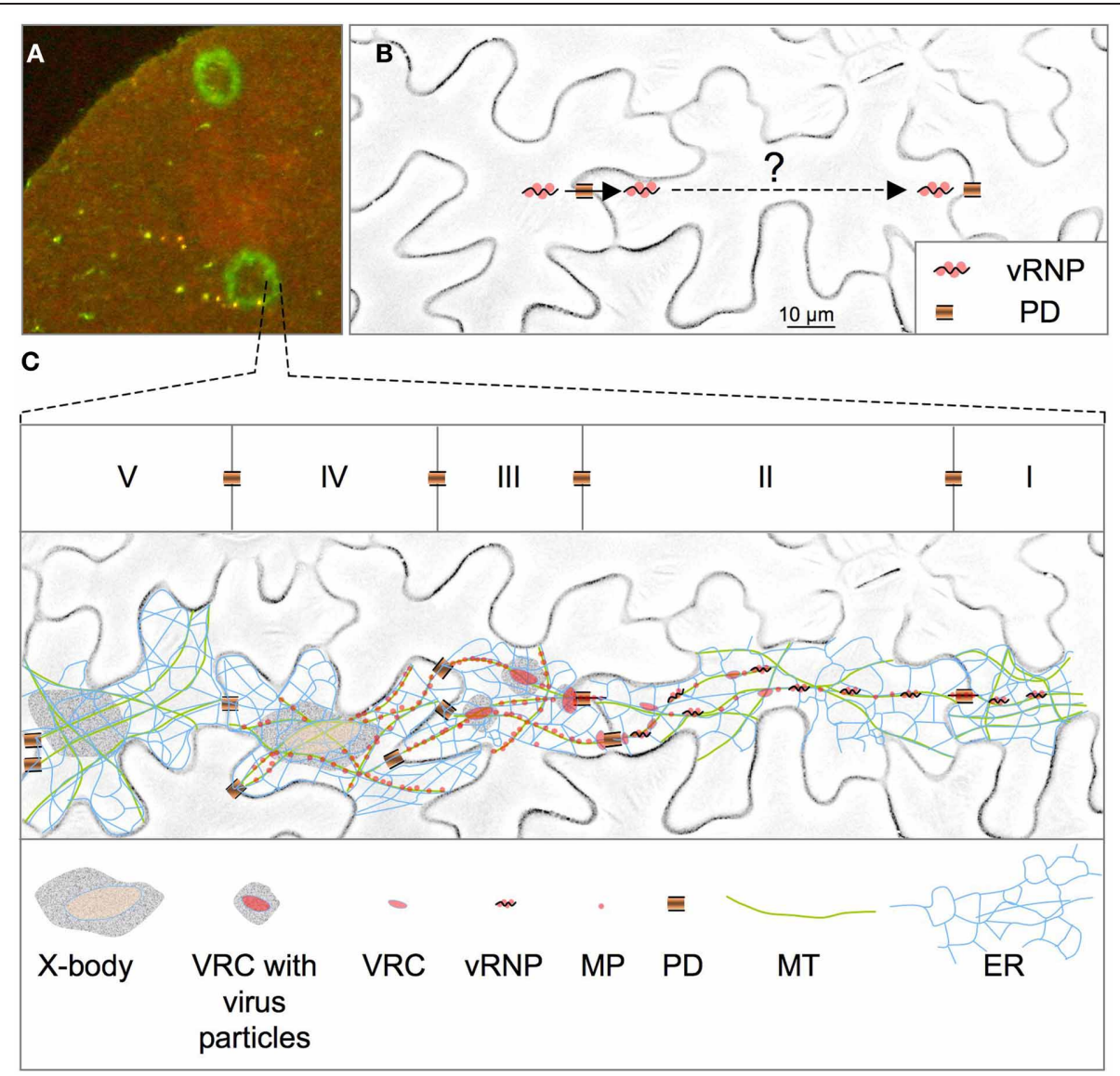

FIGURE 1 | Spreading of TMV infection. (A) Section of a Nicotiana benthamiana leaf showing infection sites of TMV-MP:GFP at 5 days post-infection. The outer rim of the infection site represents the leading front of the spreading virus. The infection site appears as a fluorescent ring because MP:GFP accumulates only transiently and is then degraded. (B) Upon entering a cell through plasmodesmata (PD) the vRNP must be replicated and transported across the diameter of the cell before it can infect the next cell. N. benthamiana epidermal cells are shown.

(C) Current model for TMV movement and the transient activities of the MP during this process. The model is projected on the pattern of five epidermal cells (depicted as I-V) at the leading front of infection. Infection of a cell (II) starts with the association of the VRNP with sites on the ER that concur with microtubules (MT) and allow the establishment of new viral replication complexes (VRCs). Following initial vRNA replication, some VRCs or VRC subcomplexes (containing vRNPs) are detached from their anchorage sites and transported via the ER through PD to infect a new cell (I). As infection already spreads, other initial VRCs continue translation and replication and increase in size over time and finally produce virus progeny (III-V). MP produced in cells behind the leading front accumulates in the MP-producing VRCs and subsequently along MT before degradation (III-IV). At late stages of infection, VRCs cease to produce MP but may continue replication to produce virion particles (IV). In a final stage all MP has disappeared (except from PD where MP is stable) and cells contain "X-bodies" with surrounding virion particles (V). provides a direct pathway for guiding the vRNP/VRC from replication sites into adjacent cells. Consistent with transport along the $\mathrm{ER}$, the MP has predicted transmembrane domains essential for membrane association and virus movement (Fujiki et al., 2006). Transport along the ER is facilitated by the ER-associated actin system and can be blocked by overexpression of actin-binding protein (Hofmann et al., 2009) (Figure 2A). The same conditions also blocked the myosin-dependent transport of Golgi complexes thus indicating a role of myosin motors in vRNP/VRC trafficking. These motors may act in vRNP/VRC transport through binding the vRNP/VRC as a transport cargo, or indirectly, by supporting bulk flow macromolecular trafficking in the membrane (Figure 2A) (Hofmann et al., 2009). However, at present it remains unclear whether vRNP/VRC transport along the ER indeed depends on the ER-associated actin system or whether the
ER alone could provide sufficient membrane-associated motility for virus movement. The latter proposal is suggested by reports showing that ER membranes maintain lateral macromolecular transport upon actin disruption or myosin inhibition, albeit with reduced efficiency (Runions et al., 2006; Griffing, 2010). Although the disruption of actin should reduce the efficiency of vRNP particle/VRC transport along the membrane, the intercellular spread of infection was not immediately inhibited upon actin disruption for $24 \mathrm{~h}$, as was seen by the uninterrupted expansion of infection sites in leaves under these conditions (Hofmann et al., 2009). However, this observation may be expected given that the movement of only few genome units is sufficient for the spread of infection (Li and Roossinck, 2004; Gutierrez et al., 2010). Nevertheless, inhibition of virus movement at the level of infection sites was observed upon long-term inhibition of the actin-myosin system 


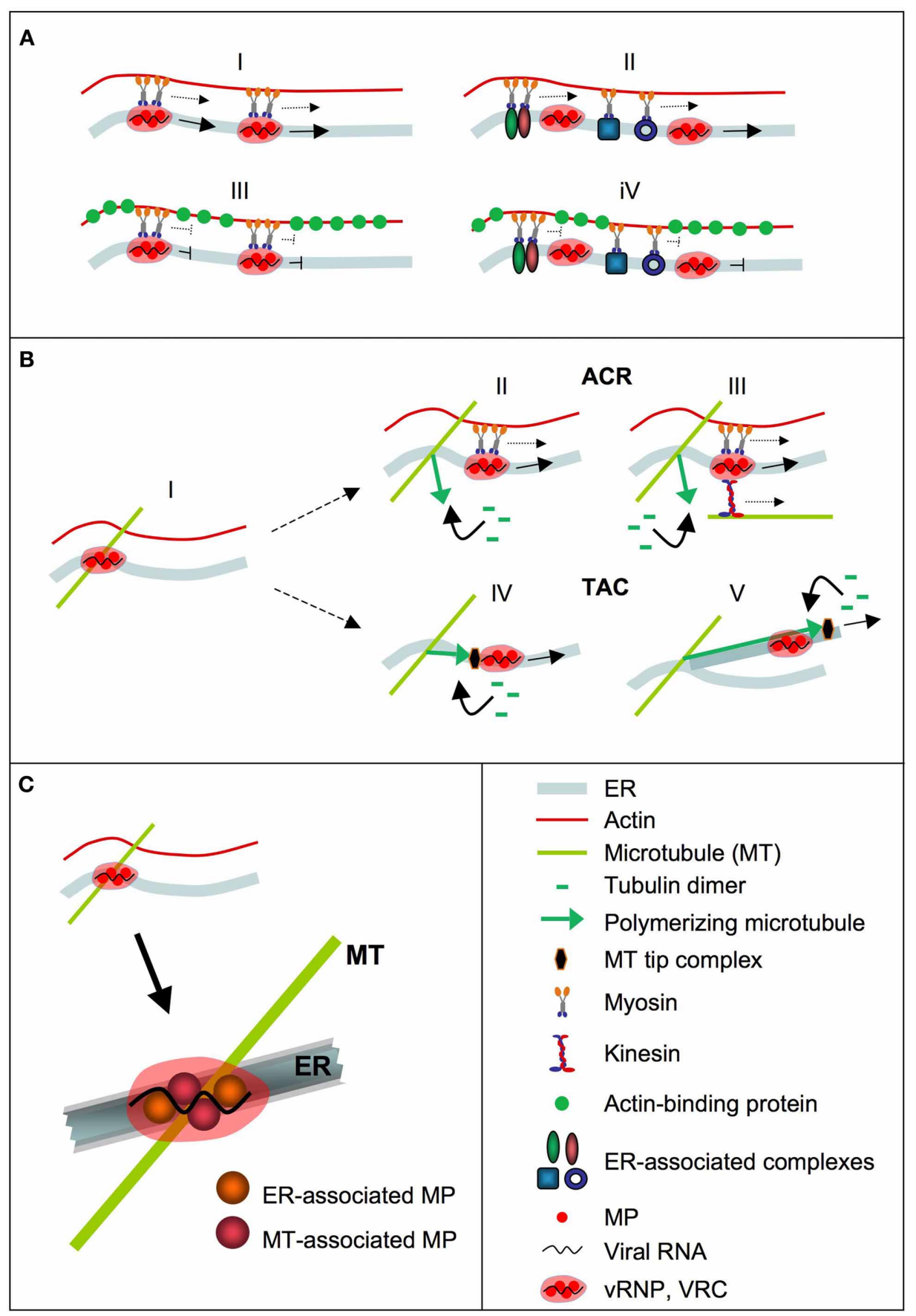

FIGURE 2 | Mechanisms in ER-mediated vRNA transport. vRNA is depicted in the form of a VRC. (A) Role of the ER-associated actin cytoskeleton in VRC transport. ER-associated VRCs may represent cargo for myosins that transport the VRCs along the ER in an actin-dependent manner (I). Transport of VRCs along the ER may also be facilitated by myosin-driven macromolecular mass flow along the membrane (II). Overexpression of actin-binding protein interferes with myosin movements along actin filaments and therefore with myosin-supported VRC transport along the membrane (III and IV). Disruption of actin interferes with the ability of myosins to facilitate directional transport but does not completely abolish VRC transport along the membrane (not shown). (B) Role of microtubules (MT) in
ER-associated VRC transport. VRCs attached at ER/MT intersections (I). In the Attachment Complex Release (ACR) mechanism, locally induced MT polymerization releases the VRCs from attachment sites for ER-guided transport (I) with support by myosins (II), kinesins, or both (III). In the Tip-Attachment Complex (TAC) mechanism, locally induced MT polymerization causes VRC detachment and also provides the force to accelerate $\mathrm{VRC}$ movement along the $\mathrm{ER}$, ether by pushing the $\mathrm{VRC}$ along existing membrane tubules (IV), or by extending ER tubules carrying the VCR (V). (C) vRNPs (VRCs) may represent higher order structures in which MP molecules with different folds that expose domains for interaction with the $\mathrm{ER}$ or MT, respectively, are combined with $\mathrm{VRNA}$ and other viral and host factors. 
( $\geq 3$ days), either by exposure to actin or myosin antagonists, or by silencing actin or myosin expression (Kawakami et al., 2004; Liu et al., 2005; Harries et al., 2009a,b). Yet, in this case, it remains to be seen whether this result implies a direct role of actin and myosin in VRC transport along the ER membrane or whether this observation could also be explained by indirect effects, for example by compromising the role of the actin cytoskeleton in maintaining the dynamic structural integrity of the ER (Wright et al., 2007; Hofmann et al., 2009; Sparkes et al., 2009b). It is also yet unclear whether the MP itself interacts with actin or myosin to facilitate movement. A direct interaction was suggested by early studies in fixed protoplasts showing an alignment of antibody-labeled MP to phalloidin-rhodamine labeled actin filaments (McLean et al., 1995). However, recent in vivo studies in leaves argued against this observation (Hofmann et al., 2009). An interaction of MP with actin was then recently again suggested by the finding that MP causes actin severing in vitro and that the stabilization of actin filaments with phalloidin inhibits the ability of MP to increase the SEL of PD in vivo (Su et al., 2010). Although these observations provide compelling evidence for a role of actin severing in the regulation of the PD SEL, it still remains uncertain whether MP interacts with actin in vivo and whether its actin severing activity would occur at PD. Recent studies indicate that the virus and its VRCs interact with actin filaments via replicase and that both replicase and actin filaments play a role in VRC formation and growth (Liu et al., 2005). Possibly, interactions between replicase and actin filaments play a role also in supporting the efficiency of vRNP/VRC movement along the ER.

\section{vRNA TRANSPORT IS CONTROLLED BY THE MICROTUBULE SYSTEM}

While further studies are needed to decipher the interactions of $\mathrm{MP}$ with the actin cytoskeleton, there is compelling evidence for the binding of MP to microtubules, tubulin, and microtubuleassociated factors (Heinlein et al., 1995; McLean et al., 1995; Padgett et al., 1996; Heinlein et al., 1998; Boyko et al., 2000a,b; Kragler et al., 2003; Ashby et al., 2006; Ferralli et al., 2006; Brandner et al., 2008; Sambade et al., 2008). MP binding to microtubules stabilizes them against disruption by polymerization inhibitors, salt, and cold (Boyko et al., 2000a; Ashby et al., 2006; Ferralli et al., 2006). The MP also has the capacity to induce changes in the microtubule array through interaction with microtubule nucleation complexes (Ferralli et al., 2006), a finding supported by the ability of MP to interact with microtubule assembly factors, such as GFP-fused EB1 (Brandner et al., 2008) and $\gamma$-tubulin (Sambade et al., 2008). In vivo functional studies correlated the interaction between MP and microtubules with MP function in virus movement (Boyko et al., 2000a,c, 2007). However, it is still unclear whether the interaction of MP with microtubules is a requirement or rather a consequence of function. Nevertheless, with respect to interactions of MP with microtubules it is important to differentiate between early and late stages of infection. During early stages of infection, thus in cells at the front of spreading infection sites in leaves, the protein interacts with microtubules in the form of ER-associated mobile particles, whereas at later stages, thus in cells behind the front, the protein accumulates along the filaments (Boyko et al., 2007; Sambade et al., 2008). The latter feature occurs in cells behind the spreading front of the virus and is thus dispensable for virus movement. Accumulation along microtubules interferes with MP particle transport (Boyko et al., 2007) and may play an important role in halting the spread of vRNPs/VRCs as soon as the virus moved into new cells. This negative regulation of $\mathrm{MP}$ activity involves the microtubule-associated protein MPB2C that enhances the sequestration of MP by microtubules and may represent a central regulator in intercellular macromolecular trafficking (Kragler et al., 2003; Curin et al., 2007; Winter et al., 2007). Consistent with the notion that the movement process itself is independent of microtubule-aligned MP, infection spreads with similar efficiency if the amount of MP produced during infection is reduced and microtubule-aligned MP is only rarely observed (Heinlein et al., 1998).

\section{ANALYSIS OF MP ACTIVITY IN RNA TRANSPORT IN THE ABSENCE OF INFECTION}

To study MP activity in RNA transport, several studies employed ectopic expression since the protein maintains function under these conditions. Thus, ectopically expressed MP complements for movement of MP-deficient virus and supports the movement of other viruses (Deom et al., 1987; Meshi et al., 1987; Holt and Beachy, 1991; Cooper et al., 1995, 1996; Atabekov et al., 1999; Vogler et al., 2008). When expressed ectopically, the MP also maintains the association with cellular targets such as the ER, microtubules, and PD, even in the absence of infection (Heinlein et al., 1998; Sambade et al., 2008; Boutant et al., 2010). Interestingly, ectopically expressed MP also maintains the capacity to form mobile complexes that strongly resemble in shape, localization and motility behavior the mobile MP complexes (which may represent early VRCs) seen at the leading front of infection (Boyko et al., 2007; Sambade et al., 2008). The mobile MP complexes formed by transiently expressed MP in the absence of infection are functionally relevant since their formation is affected by conditional amino acid mutations in MP that also affect the formation of the mobile MP complexes/early VRCs and virus movement during infection in a temperaturesensitive manner (Boyko et al., 2007; Sambade et al., 2008). It appears remarkable that ectopically expressed MP forms particles that strongly resemble those formed during infection, despite that no vRNA is present. This may indicate that MP and vRNA join existing mechanisms for RNA transport during infection. The movements of the MP complexes are directional and occur in a stop-and-go manner along the ER and always also in contact with underlying microtubules (Sambade et al., 2008). Pausing of the movements occurs at microtubule sites that may act as anchorage sites at the ER and at which the mobile complexes may be assembled and controlled. In the context of infection, these sites may represent sites for attachment of VRCs that may subsequently detach again for movement, or may remain attached and grow into larger virion-producing VRCs (X-bodies). The observation of halting movements at microtubule sites is consistent with other reports indicating that cortical plant microtubules and underlying ER tubule junctions play a role in guiding and controlling the movements of large complexes, including 
RNA-containing complexes and organelles, in the cortical cytoplasm (Crowell et al., 2009; Gutierrez et al., 2009; Cai and Cresti, 2012; Hamada et al., 2012). According to the roles of the actomyosin system in supporting directional macromolecular transport along the ER (Sparkes et al., 2009a,b), the movements of the transiently expressed MP complexes along the ER are reduced in the presence of actin inhibitors (Sambade et al., 2008). Interestingly, upon application of microtubule polymerization inhibitors the complexes remained stably anchored at microtubule sites (Sambade et al., 2008). Given that MP was shown to interact with GFP-fused microtubule-tip protein EB1 and with the microtubule-organizing center component $\gamma$-tubulin, this may suggest that the movements of the MP complexes and early VRCs depend on induced microtubule polymerization (Sambade et al., 2008). A role of microtubule polymerization in TMV movement indeed appears likely since it was shown that herbicide-resistant tobacco mutants that are affected in microtubule polymerization dynamics are also compromised in their ability to support efficient virus movement (Ouko et al., 2010). We proposed two mechanisms by which microtubule polymerization could support virus movement (Sambade and Heinlein, 2009) (Figure 2B). Whereas the Attachment Complex Release (ACR) mechanism uses microtubule polymerization to release the vRNPs/VRCs from attachment/assembly sites, the Tip-Attachment Complex (TAC) mechanism uses microtubule polymerization, thus growing microtubules, to push the complexes along the ER. The TAC mechanism plays an important role in ER tubule motility (Waterman-Storer and Salmon, 1998) and may indeed provide strong pushing forces as, for example, shown by the ability of polymerizing microtubules to move whole nuclei through the cytoplasm (Zhao et al., 2012).

\section{ANALYSIS OF MP ACTIVITY IN RNA TRANSPORT AT THE LEVEL OF LABELED RNA}

As collected evidence provides indications that the in vivo observed mobile ER-associated MP-containing complexes serve as the vehicle to transport vRNA into non-infected cells (Boyko et al., 2007; Sambade et al., 2008), it is important to determine that the particles indeed contain vRNA. Several methods for the in vivo labeling of specific RNA molecules are available and have been recently reviewed (Christensen et al., 2010). Earlier studies employed in situ hybridization of biochemically fixed, TMV-infected tobacco BY-2 protoplasts with digoxigeninlabeled probe to show in separate co-staining experiments that vRNA colocalizes with MP:GFP, with immunolabeled replicase, with immunolabeled ER, and with immunolabeled microtubules. The overlapping patterns observed are consistent with the presence of vRNA in small and large VRCs, with vRNA/VRCs present in particles along microtubules, and with a role of microtubules in the cellular distribution of vRNA/VRCs (Más and Beachy, 1999, 2000). More recently, microinjection of Cy3 pre-labeled vRNA into tobacco trichome cells was used to demonstrate that the microinjected vRNA immediately forms granules, associates with the ER in a 5'CAP-dependent manner, and moves along the ER/actin network (Christensen et al., 2009). The directional movement pattern of the labeled vRNA granules in trichomes may resemble the pattern of MP particle movements in cells at the virus front in leaves. Nevertheless, it appears probable that the vRNA granules observed in these microinjection experiments differ in nature and/or composition from mobile MP particles seen upon ectopic expression of tagged MP (Sambade et al., 2008) and from MP particles observed in cells at the spreading virus front in leaves (Boyko et al., 2007). Unlike the movements of the particles tagged with transiently expressed MP, the movement of the microinjected vRNA was insensitive to treatments with microtubule polymerization inhibitors. Moreover, microinjected vRNA was incompetent for movement between cells even in transgenic, MP-expressing plants (Christensen et al., 2009). Previous evidence indicated that MP-mediated macromolecular transport requires physical association of the transported molecules with MP (Waigmann and Zambryski, 1995). Thus, although vRNA molecules move between cells if co-injected with MP (Nguyen et al., 1996), microinjected RNA may not find plant-expressed MP and thus fails to form movement-competent complexes. It is also possible that microinjected RNA preferably associates with factors for translation and replication rather than with MP. Another approach to localize vRNA in vivo involves the use of Pumilio, an RNA binding protein, coupled to bimolecular fluorescence complementation (BiFC) (Tilsner et al., 2009). Upon introduction into infected $N$. benthamiana cells this system revealed the localization of vRNA in large VRCs and in smaller discrete particles throughout the peripheral cytoplasm. The Pumilio detection system is less invasive than microinjection. However, its application appears to be associated with a certain level of background caused by unspecific BiFC and promiscuity of Pumilio binding to RNA (Tilsner et al., 2009). In our own efforts, we have applied the non-invasive RNA detection system based on the MS2 phage CP (Bertrand et al., 1998). Expression of this protein fused to a reporter such as GFP allows specific detection of RNA molecules carrying stem-loop sequences derived from the origin of assembly (OAS) of the phage. To reduce the background of MS2-CP:GFP (MCP), the protein is fused to nuclear localization signal (NLS) so that the protein localizes to the nucleus and is retained in the cytoplasm only if associated with the RNA binding target. Our attempts to localize vRNA were not successful so far since the insertion of the MS2 OAS sequences into the vRNA interferes with TMV infectivity. However, we successfully used this system to detect the mRNA of ectopically expressed MP (Sambade et al., 2008). As described above, ectopically expressed MP:GFP is functional and forms mobile, ER-associated particles that are functionally related to the MP particles expressed in cells at the leading front of spreading infection sites in leaves. Co-expression of MP:RFP with MCP allowed the detection of mobile MP:RFP mRNA signal that coincides with mobile MP:RFP particles (Figures 3A-C). Detection of the mRNA is highly specific and depends on the presence of the OAS stem-loops in the mRNA sequence (Sambade et al., 2008). These observations revealed that the mobile complexes formed by transiently expressed MP on the ER contain MP mRNA. Moreover, MP:RFP mRNA was detected at PD (Figures 3D-I) in a manner dependent on MP (Sambade et al., 2008). However, because of the fast tracking motions and the low level of MS2-CP:GFP labeling the further analysis of the mobile RNA complexes is 

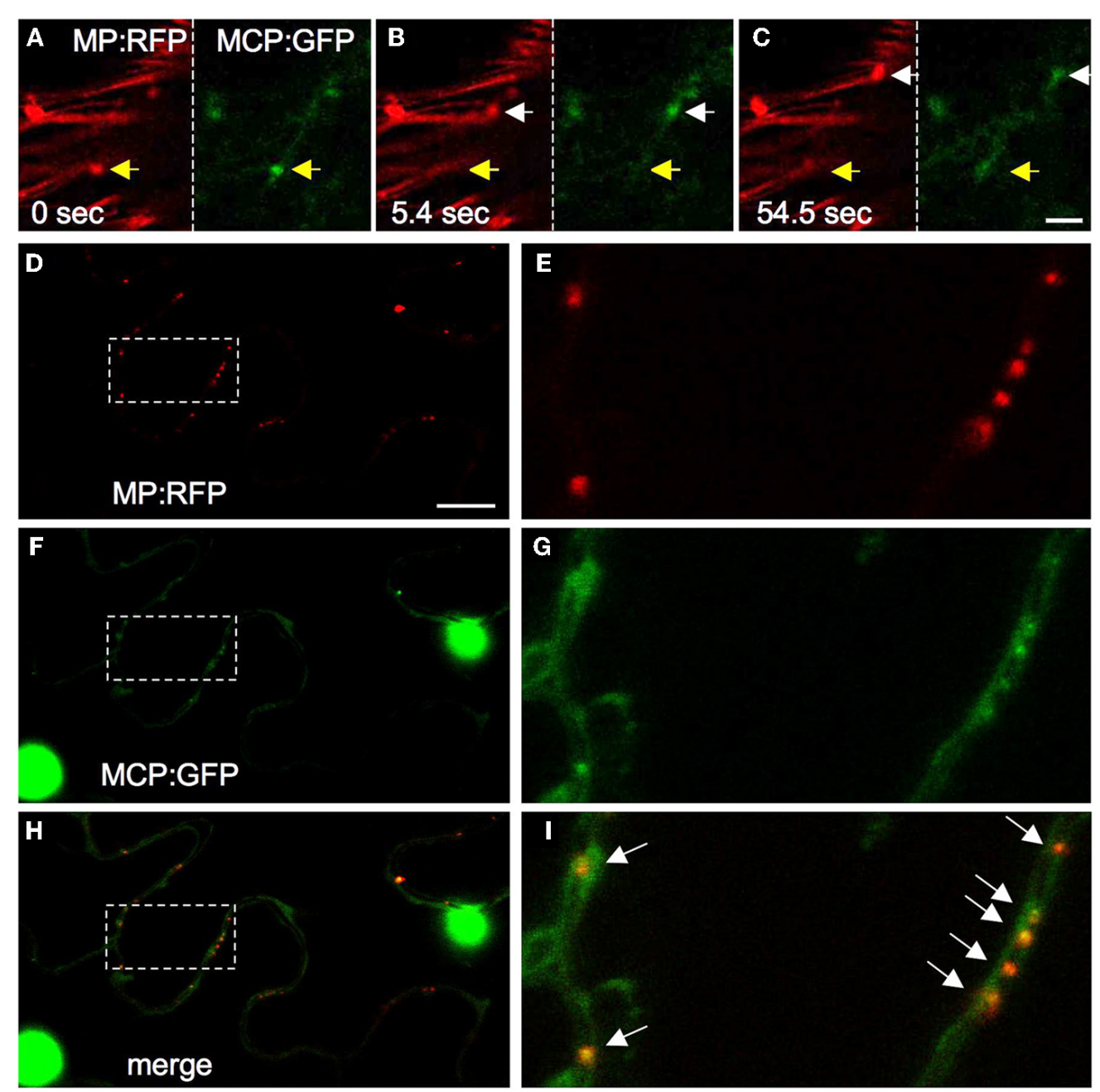

FIGURE 3 | Detection of MP mRNA with MCP. (A-C) Examples of video frames showing the coinciding movements of MP:RFP (red) and MP:RFP mRNA (green, labeled with MCP:GFP) particles. Size bar, $5 \mu \mathrm{m}$. (D-I) MP:RFP (D and E, red) and MP:RFP mRNA (F and $\mathbf{G}$, green, labeled with

MCP:GFP) coincide at the cell wall, presumably in PD (arrows in I). $\mathbf{H}$ and $\mathbf{I}$ are merged images of $\mathbf{D}$ and $\mathbf{F}$, and $\mathbf{E}$ and $\mathbf{G}$, respectively. $\mathbf{E}, \mathbf{G}$, and $\mathbf{I}$ show enlargements of the area highlighted in $\mathbf{D}, \mathbf{F}$, and $\mathbf{H}$. Size bar, $10 \mu \mathrm{m}$.

difficult. Increasing MCP expression to improve signal strength causes considerable background labeling that can strongly interfere with the interpretation of the RNA labeling results. While the MS2 system is further developed (Wu et al., 2012), our current efforts concentrate on testing the application of other RNA binding protein-dependent RNA-labeling systems, such as $\lambda \mathrm{N}$ (Daigle and Ellenberg, 2007). New approaches also include the development of sequence-specific RNA detection using aptamerbinding dyes (Lux et al., 2012). We hope that these new attempts will allow us provide further information about the composition of the MP-tagged mRNA particles and the pathway that guides them to PD.

\section{OUTLOOK}

Despite important insights into the interactions of MP with host cell components have been achieved we are still far away from understanding the molecular mechanism by which this protein facilitates the transport of vRNA between cells. More efforts are needed to reliably detect RNA in vivo and to study cell-to-cell transport at the RNA level. Further studies will also have to focus on the analysis of the composition of the MP particles. The MP particles seen upon transient expression may differ in composition from those formed at the leading front of infection as there is evidence that the viral replicase contributes to virus movement (Hirashima and Watanabe, 2001; Guenoune-Gelbart et al., 2008) and that the virus moves as a VRC (Kawakami et al., 2004). However, identifying the composition of the particles formed by transiently expressed MP may provide direct clues of how the MP interacts with the existing RNA transport pathway. It will be important also to determine if MP-mediated vRNA transport requires the activity of motor proteins. Although evidence for the involvement of myosin in virus movement has been provided (Harries et al., 2009a) it is unclear where exactly in the cell myosin activity 
is involved. Myosin motor activity accelerates macromolecular transport along the ER membrane (Sparkes et al., 2009b) and may also accelerate the diffusion of vRNPs/VRCs along the membrane towards and through PD. It will be important to determine whether MP or the replicase could represent cargo for specific myosins or whether myosins rather interact with other ER-localized complexes and support virus movement indirectly through macromolecular bulk flow in the membrane.

Another question concerns the range of molecules that the MP moves cell-to-cell. MPs can support the trafficking of different viruses and certain recombinant MPs, including the MP of TMV, were shown to facilitate intercellular trafficking of different viral nucleic acids prepared in vitro and co-injected into a cell, both of which is consistent with their sequence nonspecific nucleic acid binding activity (Ding, 1998; Heinlein and Epel, 2004). However, although sequence non-specific binding of nucleic acids may allow MP to facilitate the transport of any bound RNA, movement of VRC subcomplexes or of whole VRCs likely increases specificity for the transport of vRNAs, which are produced in VRCs. Thus, MP-mediated transport of RNAs other than vRNA may represent an exception. Nevertheless, the observation that MP enhances the intercellular spread of induced GFP-transgene silencing (Vogler et al., 2008) may indicate that the protein facilitates the movement of small RNA molecules. Spreading of virus and/or host-derived small RNAs at the leading front of infection by MP may induce transient changes in target gene expression and in the susceptibility of the cells for the moving virus (Amari et al., 2012). Understanding vRNA transport and virus movement may be enhanced by including the analysis of small RNA activity at and behind the virus front.

Further important insights into MP functions will also depend on determining the structure of MP. The protein may assume different structures in vivo since the protein domain involved in interactions with microtubules overlaps with predicted transmembrane domains involved in the association with membranes (Boyko et al., 2000a; Brill et al., 2004; Fujiki et al., 2006). The protein acts a dimer or multimer (Brill et al., 2004; Boutant et al., 2010) and thus may form higher-order structures that allow simultaneous exposure of different interaction domains through combining MP molecules with specific folds (Figure 2C). Since the protein is phosphorylated and ubiquitinylated in vivo (Lee and Lucas, 2001; Ashby et al., 2006) further studies may identify the roles of these post-translational modifications in directing the folding and function, and subsequent turnover of the protein.

It will also be important to determine how far the observations described for TMV and summarized herein also apply to the movement of other RNA viruses. Mechanisms involved in the cell-to-cell movement of viruses have been reviewed in several recent overview articles (Lucas, 2006; Harries and Ding, 2011; Niehl and Heinlein, 2011; Schoelz et al., 2011; Ueki and Citovsky, 2011) and show a role of membranes and the cytoskeleton as a common scheme. However, important differences certainly do exist. For example, viruses that move between cells in the form of virions must depend on molecular mechanisms other than viruses that move in a non-encapsidated form like TMV. Moreover, some viruses induce tubular transport structures inside PD, which involves the displacement of the ER from the PD channel. Thus, unlike TMV these viruses cannot rely on the ER as a structure that guides them to the pore. Here, endocytic vesicle trafficking can play a role, as indicated for nepoviruses (Amari et al., 2010). Whereas the actin cytoskeleton seems to play direct or indirect roles in the movement of many viruses there is yet limited evidence for microtubules playing a general role in virus movement. A recent study demonstrated that the triple gene block protein 1 (TGBp1) of Potato mop-to virus (PMTV) interacts with microtubules (Wright et al., 2010). The accumulation of this MP along microtubules is observed only in cells behind the infection front and the treatment of the plants with microtubule-disrupting agents does not interfere with further lesion growth. Although this shows that a wholly intact microtubule cytoskeleton is not required for virus movement, the TGB1 may still interact with the microtubule system in the presence of inhibitors and form microtubule-interacting structures also in cells at the virus front as was shown for TMV (Seemanpillai et al., 2006; Boyko et al., 2007). The detection of mobile RNA particles in cells at the virus front and evidence supporting a functional role of these particles in virus cell-to-cell movement is an achievement so far unique for TMV. Certainly, further studies are needed to reveal whether these observations reflect a general mechanism also applying to other viruses and RNA transport processes in plants.

However, a role of RNA-enriched mobile particles in the transport of RNA viruses and other RNA molecules is certainly consistent with similar observations in other systems. These include neuronal granules for transport of mRNAs along axons and dendrites for localized translation at synapses (Kiebler and Bassell, 2006; Doyle and Kiebler, 2011), RNA granules in Drosophila polar development (Ferrandon et al., 1997; Hachet and Ephrussi, 2004) and RNA transport particles in budding yeast (Lange et al., 2008). The key principles of mRNA localization mechanisms in these systems have been established and many players identified (Palacios and St. Johnston, 2001; Holt and Bullock, 2009; Martin and Ephrussi, 2009; Shahbabian and Chartrand, 2012). Thus, RNA transport particles can contain several or only one mRNA (Besse et al., 2009; Macdonald, 2011; Batish et al., 2012) and their transport is usually determined by cis-acting localization elements in the $3^{\prime} \mathrm{UTR}$ of the mRNAs. These elements are recognized by specific families of trans-acting RNA-binding proteins and the set of proteins that binds to the mRNA already during and after transcription in the nucleus plays a role in determining the ultimate location and fate of the mRNA within the cytoplasm. An important concept is that the mRNAs are translationally repressed during transport, and recent studies have suggested a role of noncoding RNAs and miRNAs in the transport particles (Besse and Ephrussi, 2008). The particles are transported by motor proteins (myosins, kinesins, and dyneins) along microtubules and microfilaments and their anchorage at their final destinations depends in many cases on actin. Collectively, the targeted delivery of RNA molecules in the form of particles, the presence of trans-acting proteins binding to cis-acting RNA elements, and the role of the cytoskeleton are characteristics reminiscent of those of the observed MP particles, i.e., their RNA content, the ability of MP 
to bind RNA, translational inhibition during transport (Karpova et al., 1997, 1999), and the role of the cytoskeleton. However, whether the composition of the RNA particles formed or used by MP and the mechanism that transports them along the ER have indeed any similarity with the RNA particles in other systems remains to be seen.

\section{REFERENCES}

Amari, K., Boutant, E., Hofmann, C., Schmitt-Keichinger, C., FernandezCalvino, L., Didier, P., Lerich, A., Mutterer, J., Thomas, C. L., Heinlein, M., Mely, Y., Maule, A. J., and Ritzenthaler, C. (2010). A family of plasmodesmal proteins with receptor-like properties for plant viral movement proteins. PLoS Pathog. 6:e1001119. doi: 10.1371/journal.ppat.1001119

Amari, K., Vazquez, F., and Heinlein, M. (2012). Manipulation of plant host susceptibility: an emerging role for viral movement proteins? Front. Plant Sci. 3:10. doi: 10.3389/fpls.2012.00010

Ashby, J., Boutant, E., Seemanpillai, M., Groner, A., Sambade, A., Ritzenthaler, C., and Heinlein, M. (2006). Tobacco mosaic virus movement protein functions as a structural microtubule-associated protein. J. Virol. 80, 8329-8344.

Atabekov, J. G., Malyshenko, S. I., Morozov, S. Y., Taliansky, M. E., Solovyev, A. G., Agranovsky, A. A., and Shapka, N. A. (1999). Identification and study of Tobacco mosaic virus movement function by complementation tests. Philos. Trans. R. Soc. Lond. B Biol. Sci. 354, 629-635.

Batish, M., van den Bogaard, P., Kramer, F. R., and Tyagi, S. (2012). Neuronal mRNAs travel singly into dendrites. Proc. Natl. Acad. Sci. U.S.A. 109, 4645-4650.

Bertrand, E., Chartrand, P., Schaefer, M., Shenoy, S. M., Singer, R. H., and Long, R. M. (1998). Localization of ASH1 mRNA particles in living yeast. Mol. Cell 2, 437-445.

Besse, F., De Quinto, S. L., Marchand, V., Trucco, A., and Ephrussi, A. (2009). Drosophila PTB promotes formation of high-order RNP particles and represses oskar translation. Genes Dev. 23, 195-207.

Besse, F., and Ephrussi, A. (2008). Translational control of localized mRNAs: restricting protein synthesis in space and time. Nat. Rev. Mol. Cell Biol. 9, 971-980.

Boutant, E., Didier, P., Niehl, A., Mély, Y., Ritzenthaler, C., and Heinlein, M. (2010). Fluorescent protein recruitment assay for demonstration and analysis of in vivo protein interactions in plant cells and its application to Tobacco mosaic virus movement protein. Plant J. 62, 171-177.

Boyko, V., Ferralli, J., Ashby, J., Schellenbaum, P., and Heinlein, M. (2000a). Function of microtubules in intercellular transport of plant virus RNA. Nat. Cell Biol. 2, 826-832.

Boyko, V., Ferralli, J., and Heinlein, M. (2000b). Cell-to-cell movement of TMV RNA is temperaturedependent and corresponds to the association of movement protein with microtubules. Plant J. 22, 315-325.

Boyko, V., van der Laak, J., Ferralli, J., Suslova, E., Kwon, M.-O., and Heinlein, M. (2000c). Cellular targets of functional and dysfunctional mutants of Tobacco mosaic virus movement protein fused to GFP. J. Virol. 74, 11339-11346.

Boyko, V., Hu, Q., Seemanpillai, M., Ashby, J., and Heinlein, M. (2007). Validation of microtubuleassociated Tobacco mosaic virus RNA movement and involvement of microtubule-aligned particle trafficking. Plant J. 51, 589-603.

Brandner, K., Sambade, A., Boutant, E., Didier, P., Mély, Y., Ritzenthaler, C., and Heinlein, M. (2008). Tobacco mosaic virus movement protein interacts with green fluorescent protein-tagged microtubule endbinding protein 1. Plant Physiol. 147, 611-623.

Brill, L. M., Dechongkit, S., Delabarre, B., Stroebel, J., Beachy, R. N., and Yeager, M. (2004). Dimerization of recombinant Tobacco mosaic virus movement protein. J. Virol. 78, 3372-3377.

Cai, G., and Cresti, M. (2012). Are kinesins required for organelle trafficking in plant cells? Front. Plant Sci. 3:170. doi: 10.3389/fpls.2012.00170

Christensen, N., Tilsner, J., Bell, K., Hammann, P., Parton, R., Lacomme, C., and Oparka, K. (2009). The $5^{\prime}$ cap of Tobacco mosaic virus (TMV) is required for virion attachment to the actin/ER network during early infection. Traffic 10, 536-551.

\section{ACKNOWLEDGMENTS}

The authors acknowledge financial funding provided by the Agence Nationale de la Recherche (ANR-08-BLAN-0244) and the Swiss National Science Foundation (31003A_124940). The authors also thank Annette Niehl for her comments during the preparation of this manuscript.

Christensen, N. M., Oparka, K. J., and Tilsner, J. (2010). Advances in imaging RNA in plants. Trends Plant Sci. 15, 196-203.

Citovsky, V. (1999). Tobacco mosaic virus: a pioneer of cell-to-cell movement. Philos. Trans. R. Soc. Lond. B Biol. Sci. 354, 637-643.

Citovsky, V., Knorr, D., Schuster, G. and Zambryski, P. (1990). The P30 movement protein of Tobacco mosaic virus is a single-stranded nucleic acid binding protein. Cell 60, 637-647.

Cooper, B., Lapidot, M., Heick, J. A., Dodds, J. A., and Beachy, R. N. (1995). A defective movement protein of TMV in transgenic plants confers resistance to multiple viruses whereas the functional ana$\log$ increases susceptibility. Virology 206, 307-313.

Cooper, B., Schmitz, I., Rao, A. L., Beachy, R. N., and Dodds, J. A. (1996). Cell-to-cell transport of movement-defective cucumber mosaic and tobacco mosaic viruses in transgenic plants expressing heterologous movement protein genes. Virology 216, 208-213.

Crowell, E. F., Bischoff, V., Desprez, T., Rolland, A., Stierhof, Y. D., Schumacher, K., Gonneau, M., Höfte, H., and Vernhettes, S. (2009). Pausing of Golgi bodies on microtubules regulates secretion of cellulose synthase complexes in Arabidopsis. Plant Cell 21, 1141-1154.

Curin, M., Ojangu, E. L., Trutnyeva, K., Ilau, B., Truve, E., and Waigmann, E. (2007). MPB2C, a microtubuleassociated plant factor, is required for microtubular accumulation of Tobacco mosaic virus movement protein in plants. Plant Physiol. 143, 801-811.

Daigle, N., and Ellenberg, J. (2007). $\lambda N-G F P$ : an RNA reporter system for live-cell imaging. Nat. Methods 4, 633-636.

Deom, C. M., Oliver, M. J., and Beachy, R. N. (1987). The 30-kilodalton gene product of Tobacco mosaic virus potentiates virus movement. Science 237, 384-389.

Ding, B. (1998). Intercellular protein trafficking through plasmodesmata. Plant Mol. Biol. 38, 279-310.
Doyle, M., and Kiebler, M. A. (2011). Mechanisms of dendritic mRNA transport and its role in synaptic tagging. EMBO J. 30, 3540-3552.

Ferralli, J., Ashby, J., Fasler, M., Boyko, V., and Heinlein, M. (2006) Disruption of microtubule organization and centrosome function by expression of Tobacco mosaic virus movement protein. J. Virol. 80, 5807-5821.

Ferrandon, D., Koch, I., Westhof, E. and Nusslein-Volhard, C. (1997). RNA-RNA interaction is required for the formation of specific bicoid mRNA 3' UTR-STAUFEN ribonucleoprotein particles. EMBO J. 16, 1751-1758.

Fujiki, M., Kawakami, S., Kim, R. W., and Beachy, R. N. (2006). Domains of Tobacco mosaic virus movement protein essential for its membrane association. J. Gen. Virol. 87, 2699-2707.

Griffing, L. R. (2010). Networking in the endoplasmic reticulum. Biochem. Soc. Trans. 38, 747-753.

Guenoune-Gelbart, D., Elbaum, M., Sagi, G., Levy, A., and Epel, B. L. (2008). Tobacco mosaic virus (TMV) replicase and movement protein function synergistically in facilitating TMV spread by lateral diffusion in the plasmodesmal desmotubule of Nicotiana benthamiana Mol. Plant Microbe Interact. 21, 335-345.

Gutierrez, R., Lindeboom, J. J., Paredez, A. R., Emons, A. M., and Ehrhardt, D. W. (2009). Arabidopsis cortical microtubules position cellulose synthase delivery to the plasma membrane and interact with cellulose synthase trafficking compartments. Nat. Cell Biol. 11, 797-806.

Gutierrez, S., Yvon, M., Thebaud, G., Monsion, B., Michalakis, Y., and Blanc, S. (2010). Dynamics of the multiplicity of cellular infection in a plant virus. PLoS Pathog. 6:e1001113. doi: 10.1371/journal.ppat.1001113

Hachet, O., and Ephrussi, A. (2004). Splicing of oskar RNA in the nucleus is coupled to its cytoplasmic localization. Nature 428, 959-963.

Hamada, T., Tominaga, M., Fukaya, T., Nakamura, M., Nakano, A., Watanabe, Y., Hashimoto, T., 
and Baskin, T. I. (2012). RNA processing bodies, peroxisomes, Golgi bodies, mitochondria, and endoplasmic reticulum tubule junctions frequently pause at cortical microtubules. Plant Cell Physiol. 53, 699-708.

Harries, P., and Ding, B. (2011). Cellular factors in plant virus movement: at the leading edge of macromolecular trafficking in plants. Virology 411, 237-243.

Harries, P. A., Park, J. W., Sasaki, N., Ballard, K. D., Maule, A. J., and Nelson, R. S. (2009a). Differing requirements for actin and myosin by plant viruses for sustained intercellular movement. Proc. Natl. Acad. Sci. U.S.A. 106, 17594-17599.

Harries, P. A., Schoelz, J. E., and Nelson, R. S. (2009b). Covering common ground: F-actin-dependent transport of plant viral protein inclusions reveals a novel mechanism for movement utilized by unrelated viral proteins. Plant Signal. Behav. 4, 454-456.

Heinlein, M. (2002). Plasmodesmata: dynamic regulation and role in macromolecular cell-to-cell signaling. Curr. Opin. Plant Biol. 5, 543-552.

Heinlein, M., Epel, B. L., Padgett, H. S., and Beachy, R. N. (1995). Interaction of tobamovirus movement proteins with the plant cytoskeleton. Science 270, 1983-1985.

Heinlein, M., and Epel, B. L. (2004). Macromolecular transport and signaling through plasmodesmata. Int. Rev. Cytol. 235, 93-164.

Heinlein, M., Padgett, H. S., Gens, J. S., Pickard, B. G., Casper, S. J., Epel, B. L., and Beachy, R. N. (1998). Changing patterns of localization of the Tobacco mosaic virus movement protein and replicase to the endoplasmic reticulum and microtubules during infection. Plant Cell 10, 1107-1120.

Hirashima, K., and Watanabe, Y. (2001). Tobamovirus replicase coding region is involved in cell-to-cell movement. J. Virol. 75, 8831-8836.

Hofmann, C., Niehl, A., Sambade, A., Steinmetz, A., and Heinlein, M. (2009). Inhibition of TMV movement by expression of an actinbinding protein. Plant Physiol. 149, 1810-1823.

Holt, C. A., and Beachy, R. N. (1991). In vivo complementation of infectious transcripts from mutant Tobacco mosaic virus cDNAs in transgenic plants. Virology 181, 109-117.

Holt, C. E., and Bullock, S. L. (2009). Subcellular mRNA localization in animal cells and why it matters. Science 326, 1212-1216.

Karpova, O. V., Ivanov, K. I., Rodionova, P., Dorokhov, Y. L., and Atabekov, J. G. (1997). Nontranslatability and dissimilar behavior in plants and protoplasts of viral RNA and movement protein complexes formed in vitro. Virology 230, 11-21.

Karpova, O. V., Rodionova, N. P., Ivanov, K. I., Kozlovsky, S. V., Dorokhov, Y. L., and Atabekov, J. G. (1999). Phosphorylation of Tobacco mosaic virus movement protein abolishes its translation repressing ability. Virology 261, 20-24.

Kawakami, S., Watanabe, Y., and Beachy, R. N. (2004). Tobacco mosaic virus infection spreads cell to cell as intact replication complexes. Proc. Natl. Acad. Sci. U.S.A. 101, 6291-6296.

Kiebler, M. A., and Bassell, G. J. (2006). Neuronal RNA granules: movers and makers. Neuron 51, 685-690.

Kotlizky, G., Katz, A., van der Laak, J., Boyko, V., Lapidot, M., Beachy, R. N., Heinlein, M., and Epel, B. L. (2001). A dysfunctional movement protein of Tobacco mosaic virus interferes with targeting of wildtype movement protein to microtubules. Mol. Plant Microbe Interact. 14, 895-904.

Kragler, F., Curin, M., Trutnyeva, K., Gansch, A., and Waigmann, E. (2003). MPB2C, a microtubuleassociated plant protein binds to and interferes with cell-to-cell transport of Tobacco mosaic virus movement protein. Plant Physiol. 132, 1870-1883.

Lange, S., Katayama, Y., Schmid, M., Burkacky, O., Brauchle, C., Lamb, D. C., and Jansen, R. P. (2008). Simultaneous transport of different localized mRNA species revealed by live-cell imaging. Traffic 9, 1256-1267.

Lee, J. Y., and Lucas, W. J. (2001). Phosphorylation of viral movement proteins-regulation of cell-to-cell trafficking. Trends Microbiol. 9, 5-8.

Li, H., and Roossinck, M. J. (2004). Genetic bottlenecks reduce population variation in an experimental RNA virus population. J. Virol. 78, 10582-10587.

Liu, J.-Z., Blancaflor, E. B., and Nelson, R. S. (2005). The Tobacco mosaic virus 126-kilodalton protein, a constituent of the virus replication complex, alone or within the complex aligns with and traffics along microfilaments. Plant Physiol. 138, 1877-1895.

Lucas, W. J. (2006). Plant viral movement proteins: agents for cell-to-cell trafficking of viral genomes. Virology 344, 169-184.

Lux, J., Peña, E. J., Bolze, F., Heinlein M., and Nicoud, J. F. (2012). Malachite green derivatives for two-photon RNA detection. Chembiochem 13, 1206-1213.

Macdonald, P. M. (2011). mRNA localization: assembly of transport complexes and their incorporation into particles. Curr. Opin. Gen. Dev. 21, 407-413.

Martin, K. C., and Ephrussi, A. (2009). mRNA Localization: Gene expression in the spatial dimension. Cell 136, 719-730.

Maule, A. J. (2008). Plasmodesmata: structure, function and biogenesis. Curr. Opin. Plant Biol. 11, 680-686.

McLean, B. G., Zupan, J., and Zambryski, P. C. (1995). Tobacco mosaic virus movement protein associates with the cytoskeleton in tobacco plants. Plant Cell 7, 2101-2114.

Meshi, T., Watanabe, Y., Saito, T., Sugimoto, A., Maeda, T., and Okada, Y. (1987). Function of the $30 \mathrm{kd}$ protein of Tobacco mosaic virus: involvement in cell-to-cell movement and dispensability for replication. EMBO J. 6, 2557-2563.

Más, P., and Beachy, R. N. (1999). Replication of Tobacco mosaic virus on endoplasmic reticulum and role of the cytoskeleton and virus movement in intracellular distribution of viral RNA. J. Cell Biol. 147, 945-958.

Más, P., and Beachy, R. N. (2000). Role of microtubules in the intracellular distribution of Tobacco mosaic virus movement protein. Proc. Natl. Acad. Sci. U.S.A. 97, 12345-12349.

Nguyen, L., Lucas, W. J., Ding, B., and Zaitlin, M. (1996). Viral RNA trafficking is inhibited in replicasemediated resistant transgenic tobacco plants. Proc. Natl. Acad. Sci. U.S.A. 93, 12643-12647.

Niehl, A., and Heinlein, M. (2011). Cellular pathways for viral transport through plasmodesmata. Protoplasma 248, 75-99.

Oparka, K. J., Prior, D. A. M., Santa Cruz, S., Padgett, H. S., and Beachy, R. N. (1997). Gating of epidermal plasmodesmata is restricted to the leading edge of expanding infection sites of Tobacco mosaic virus. Plant J. 12, 781-789.

Ouko, M. O., Sambade, A., Brandner, K., Niehl, A., Pena, E., Ahad, A. Heinlein, M., and Nick, P. (2010). Tobacco mutants with reduced microtubule dynamics are less susceptible to TMV. Plant J. 62, 829-839.

Padgett, H. S., Epel, B. L., Kahn, T. W., Heinlein, M., Watanabe, Y., and
Beachy, R. N. (1996). Distribution of tobamovirus movement protein in infected cells and implications for cell-to-cell spread of infection. Plant J. 10, 1079-1088.

Palacios, I. M., and St. Johnston, D. (2001). Getting the message across: the intracellular localization of mRNAs in higher eukaryotes. Annu. Rev. Cell Dev. Biol. 17, 569-614.

Peña, E., Niehl, A., and Heinlein, M. (2012). "Viral studies point the way: mechanisms of intercellular transport," in Short and long distance signaling, eds F. Kragler and M. Hülskamp (Heidelberg, Germany: Springer), 1-43.

Runions, J., Brach, T., Kuhner, S., and Hawes, C. (2006). Photoactivation of GFP reveals protein dynamics within the endoplasmic reticulum membrane. J. Exp. Bot. 57, 43-50.

Sambade, A., Brandner, K., Hofmann, C., Seemanpillai, M., Mutterer, J., and Heinlein, M. (2008). Transport of TMV movement protein particles associated with the targeting of RNA to plasmodesmata. Traffic 9, 2073-2088.

Sambade, A., and Heinlein, M. (2009). Approaching the cellular mechanism that supports the intercellular spread of Tobacco mosaic virus. Plant Signal. Behav. 4, 35-38.

Schoelz, J. E., Harries, P. A., and Nelson, R. S. (2011). Intracellular transport of plant viruses: finding the door out of the cell. Mol. Plant 4, 813-831.

Seemanpillai, M., Elamawi, R., Ritzenthaler, C., and Heinlein, M. (2006). Challenging the role of microtubules in Tobacco mosaic virus movement by drug treatments is disputable. J. Virol. 80, 6712-6715.

Shahbabian, K., and Chartrand, P. (2012). Control of cytoplasmic mRNA localization. Cell. Mol. Life Sci. 69, 535-552.

Sparkes, I., Runions, J., Hawes, C., and Griffing, L. (2009a). Movement and remodeling of the endoplasmic reticulum in nondividing cells of tobacco leaves. Plant Cell 21, 3937-3949.

Sparkes, I. A., Frigerio, L., Tolley, N., and Hawes, C. (2009b). The plant endoplasmic reticulum: a cell-wide web. Biochem. J. 423, 145-155.

Su, S., Liu, Z., Chen, C., Zhang, Y., Wang, X., Zhu, L., Miao, L., Wang, X. C., and Yuan, M. (2010). Cucumber mosaic virus movement protein severs actin filaments to increase the plasmodesmal size exclusion limit in tobacco. Plant Cell 22, 1373-1387. 
Szécsi, J., Ding, X. S., Lim, C. O., Bendahmane, M., Cho, M. J., Nelson, R. S., and Beachy, R. N. (1999). Development of Tobacco mosaic virus infection sites in Nicothiana benthamiana. Mol. Plant Microbe Interact. 2, 143-152.

Tilsner, J., Linnik, O., Christensen, N. M., Bell, K., Roberts, I. M., Lacomme, C., and Oparka, K. J. (2009). Live-cell imaging of viral RNA genomes using a Pumiliobased reporter. Plant J. 57, 758-770.

Ueki, S., and Citovsky, V. (2011). To gate, or not to gate: regulatory mechanisms for intercellular protein transport and virus movement in plants. Mol. Plant 4, 782-793.

Verchot-Lubicz, J., and Goldstein, R. E. (2010). Cytoplasmic streaming enables the distribution of molecules and vesicles in large plant cells. Protoplasma 240, 99-107.

Vogler, H., Kwon, M. O., Dang, V., Sambade, A., Fasler, M., Ashby, J., and Heinlein, M. (2008). Tobacco mosaic virus movement protein enhances the spread of RNA silencing. PLoS Pathog. 4:e1000038. doi: 10.1371/journal.ppat. 1000038
Waigmann, E., Curin, M., and Heinlein, M. (2007). "Tobacco mosaic virus - a model for macromolecular cell-to-cell spread," in Viral Transport in Plants, eds E. Waigmann and M. Heinlein (Heidelberg, Germany: Springer), 29-62.

Waigmann, E., and Zambryski, P. (1995). Tobacco mosaic virus movement protein-mediated protein transport between trichome cells. Plant Cell 7, 2069-2079.

Waterman-Storer, C., and Salmon, E. D. (1998). Endoplasmic reticulum membrane tubules are distributed by microtubules in living cells using three distinct mechanisms. Curr. Biol. 8, 798-806.

Winter, N., Kollwig, G., Zhang, S., and Kragler, F. (2007). MPB2C, a microtubule-associated protein, regulates non-cell-autonomy of the homeodomain protein KNOTTED1. Plant Cell 19, 3001-3018.

Wolf, S., Deom, C. M., Beachy, R. N., and Lucas, W. J. (1989). Movement protein of Tobacco mosaic virus modifies plasmodesmatal size exclusion limit. Science 246, 377-379.

Wright, K. M., Cowan, G. H., Lukhovitskaya, N. I., Tilsner, J., Roberts, A. G., Savenkov, E. I., and Torrance, L. (2010). The N-terminal domain of PMTV TGB1 movement protein is required for nucleolar localization, microtubule association, and long-distance movement. Mol. Plant Microbe Interact. 23, 1486-1497.

Wright, K. M., Wood, N. T., Roberts, A. G., Chapman, S., Boevink, P., Mackenzie, K. M., and Oparka, K. J. (2007). Targeting of TMV movement protein to plasmodesmata requires the actin/ER network; evidence from FRAP. Traffic 8 , 21-31.

Wu, B., Chao, J. A., and Singer, R. H. (2012). Fluorescence fluctuation spectroscopy enables quantitative imaging of single mRNAs in living cells. Biophys. J. 102, 2936-2944.

Zhao, T., Graham, O. S., Raposo, A., and St Johnston, D. (2012). Growing microtubules push the oocyte nucleus to polarize the
Drosophila dorsal-ventral axis. Science 336, 999-1003.

Conflict of Interest Statement: The authors declare that the research was conducted in the absence of any commercial or financial relationships that could be construed as a potential conflict of interest.

Received: 05 July 2012; paper pending published: 25 July 2012; accepted: 06 August 2012; published online: 28 August 2012.

Citation: Peña EJ and Heinlein M (2012) RNA transport during TMV cell-to-cell movement. Front. Plant Sci. 3:193. doi: 10.3389/fpls.2012.00193

This article was submitted to Frontiers in Plant Physiology, a specialty of Frontiers in Plant Science.

Copyright (c) 2012 Peña and Heinlein. This is an open-access article distributed under the terms of the Creative Commons Attribution License, which permits use, distribution and reproduction in other forums, provided the original authors and source are credited and subject to any copyright notices concerning any third-party graphics etc. 